\title{
The solar wind in time
}

\author{
Jeffrey L. Linsky ${ }^{1}$, Brian E. Wood ${ }^{2}$ and Seth Redfield ${ }^{3}$ \\ ${ }^{1}$ JILA, University of Colorado and NIST, \\ Boulder, Colorado 80309-0440, USA \\ email: jlinsky@jilau1.colorado.edu \\ ${ }^{2}$ Naval Research Laboratory, Space Science Division, \\ Washington, DC 20375, USA \\ email: brian. wood@nrl.navy.mil \\ ${ }^{3}$ Astronomy Department, Wesleyan University, Middletown, CT 06459, USA \\ email: sredfield@wesleyan.edu
}

\begin{abstract}
We describe our method for measuring mass loss rates of $\mathrm{F}-\mathrm{M}$ main sequence stars with high-resolution Lyman- $\alpha$ line profiles. Our diagnostic is the extra absorption on the blue side the interstellar hydrogen absorption produced by neutral hydrogen gas in the hydrogen walls of stars. For stars with low X-ray fluxes, the correlation of observed mass loss rate with $\mathrm{X}$-ray surface flux and age predicts the solar wind mass flux between $700 \mathrm{Myr}$ and the present.
\end{abstract}

Keywords. ISM:lines and bands, ISM:magnetic fields, solar wind, stars:winds, Sun: UV radiation, ultraviolet:ISM

\section{Why mass loss rates are needed for the Sun and cool dwarf stars}

Ultraviolet spectroscopy is an important tool for measuring mass loss rates $(\dot{\mathrm{M}})$ of massive stars, late-type giants, and premain sequence stars when $\dot{\mathrm{M}}>10^{-10} \mathrm{M}_{\odot} \mathrm{yr}^{-1}$. By comparison, the solar mass loss rate measured by space experiments is far smaller, about $2 \times 10^{-14} \mathrm{M}_{\odot} \mathrm{yr}^{-1}$. Until 10 years ago, there were no measured values of $\dot{\mathrm{M}}$ for main sequence stars cooler than about spectral type A because there were no spectral or other diagnostics capable of measuring $\dot{\mathrm{M}}$ far smaller than $10^{-10} \mathrm{M}_{\odot} \mathrm{yr}^{-1}$.

While mass loss rates similar to the Sun do not significantly reduce a star's mass and thus nuclear evolution during its lifetime (see below), accurate measurements of $\dot{M}$ are needed for many reasons: (1) the torque exerted by even weak stellar winds reduces the stellar rotation rate and thus the magnetic dynamo and stellar activity, (2) knowledge of $\dot{\mathrm{M}}(\mathrm{t})$ for the young Sun is important for understanding the evolution of the atmospheres of solar system planets like Mars that have lost their magnetic fields, and (3) the discovery of exopolanets close to their host stars highlights the need to predict the properties of stellar winds that impact the atmospheres of these exoplanets. Since we cannot measure $\dot{\mathrm{M}}_{\odot}$ back in time, it is critical to find a reliable diagnostic to measure $\dot{\mathrm{M}}$ for solar-mass stars with a range of ages and coronal properties in order to scale to the young Sun. Unfortunately, theoretical estimates of $\dot{M}$ are controversial, an empirical relationship between $\dot{\mathrm{M}}$ and X-ray flux was not previously known, and no space probe will enter an astrosphere to directly measure $\dot{M}$ for a very long time.

\section{The Lyman- $\alpha$ diagnostic for stellar mass loss rates}

Baranov \& Malama (1995) showed that charge exchange processes between solar wind protons and neutral hydrogen atoms in the local interstellar medium (ISM) produce a population of heated, decelerated hydrogen atoms concentrated in the interstellar upwind 


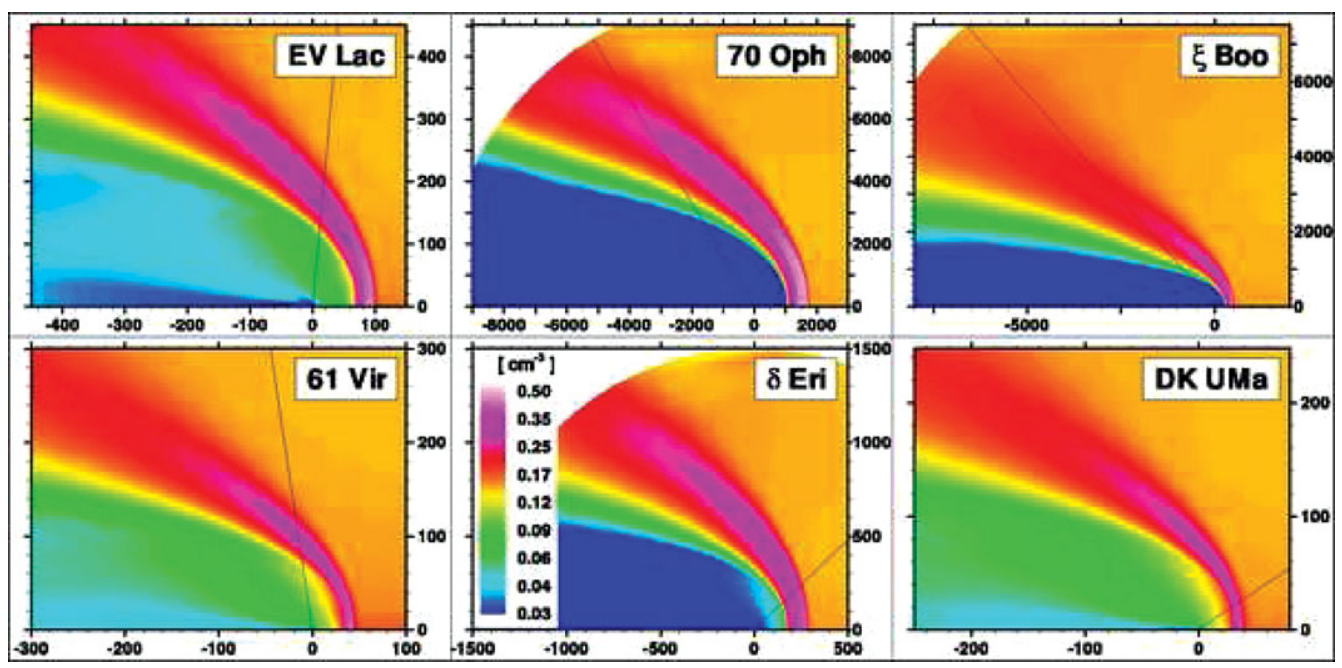

Figure 1. Theoretical models including charge exchange processes of the neutral hydrogen distribution around six stars. Units for the $\mathrm{x}$ and $\mathrm{y}$ axes are Astronomical Units, and the stars are moving to the right compared to the local ISM. High densities (red color, in the online version of this paper) indicate the location of hydrogen walls. Solid lines show the line of sight to the Earth (Wood et al. 2005).

direction, the so-called "hydrogen wall". These calculations and those in subsequent models of Zank et al. (1996), Izmodenov et al. (1999), and Müller et al. (2001) allowed Wood et al. (2001) to compute $\dot{\mathrm{M}}$ for $\alpha$ Cen. They showed that along the line of sight to the star, the stellar Lyman- $\alpha$ line contains three absorption components due to interstellar hydrogen, the solar hydrogen wall, and the stellar hydrogen wall. The decelerated hydrogen atoms in the solar hydrogen wall produce absorption on the red side of interstellar absorption. Hydrogen atoms in stellar hydrogen walls are decelerated relative to the ISM as seen by the star and thus blue-shifted relative to the interstellar absorption as seen from the Earth.

Figure 1 shows maps of neutral hydrogen density computed for 6 stars studied by Wood et al. (2005). These models show the presence of hydrogen walls around the stars. Figure 2 shows the Lyman- $\alpha$ profiles for these stars observed with the Space Telescope Imaging Spectrograph on the Hubble Space Telescope. Also included are the interstellar hydrogen absorption profiles predicted from the observed deuterium Lyman- $\alpha$ interstellar absorption and additional astrosphere absorption based on models with a range of $\dot{M}$ values relative to the solar value. These models assume that the stellar wind speed is $400 \mathrm{~km} \mathrm{~s}^{-1}$, but $\dot{\mathrm{M}}$ scales roughly linearly with the wind speed.

The effect of increasing stellar mass loss rate is to increase the absorption on the blue side of the interstellar absorption. The theoretical Lyman- $\alpha$ profiles, which have been convolved with the instrumental profile, show good agreement with the observed profiles at a best fit value of $\dot{M}$ with about a factor of 2 uncertainty.

Figure 3 (left) shows the values of $\dot{\mathrm{M}}$ obtained for 12 stars studied by Wood et al. (2005) and the Sun compared with the X-ray surface fluxes $\left(F_{X}\right)$, obtained at different times than the $\dot{M}$ measurements. Since X-ray emission is variable, there should be some scatter in any correlation of such non-simulataneous data. Nevertheless, there appears to be a correlation of $\dot{\mathrm{M}}$ per $\mathrm{cm}^{2}$ of stellar surface with $F_{X}$ over two orders of magnitude in $\dot{M}$ with the Sun consistent with the trend. The power-law fit to these data is 


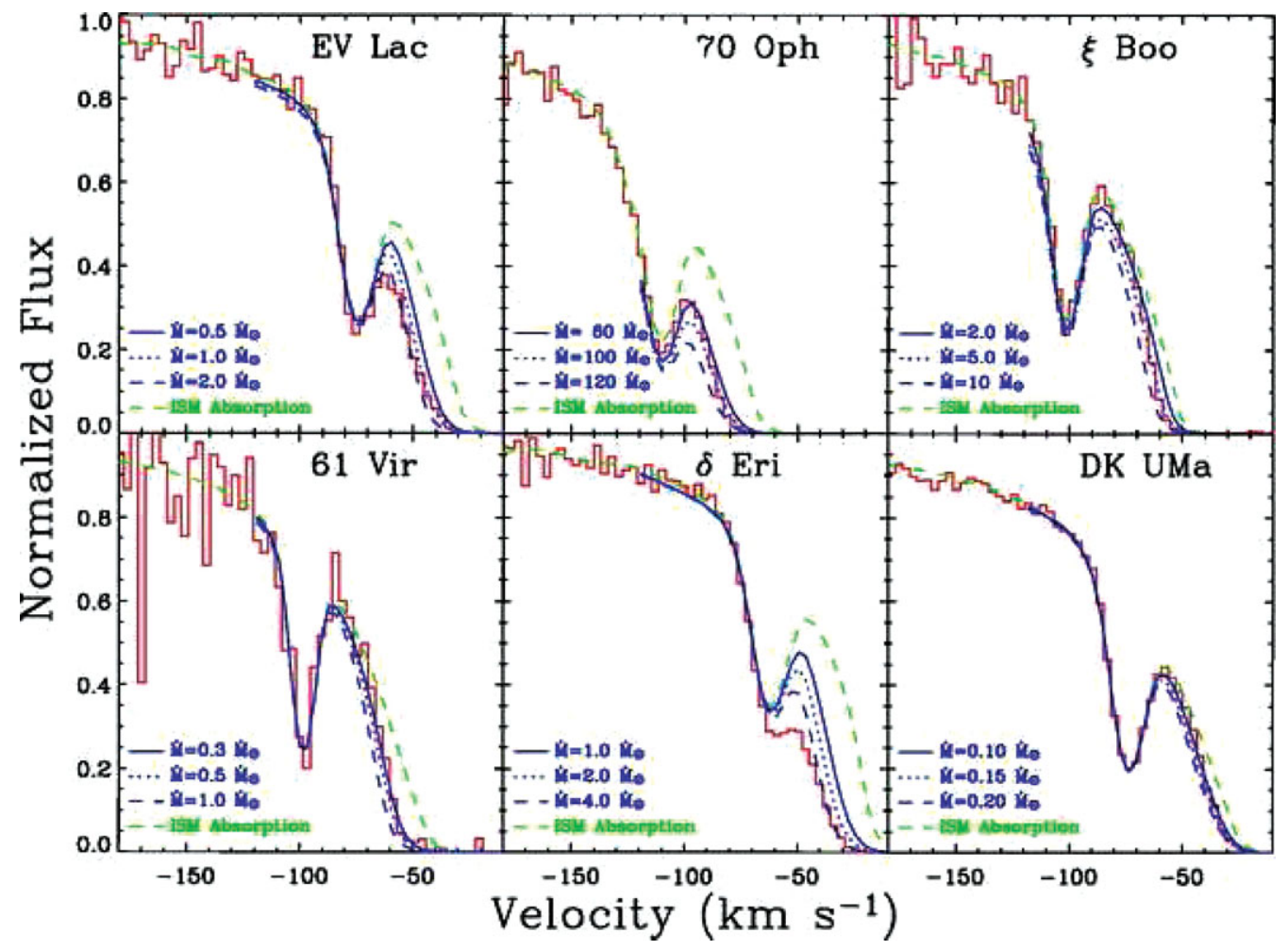

Figure 2. Observed Lyman- $\alpha$ profiles (red histograms) are compared to models with no mass loss (green (in the online version) dashed line marked ISM absorption) and models with different mass loss rates compared to the solar value (Wood et al. 2005). For a color figure, see the online version of the paper.

$$
\dot{M} \propto F_{X}^{1.34 \pm 0.18}
$$

For $F_{X}>8 \times 10^{5} \mathrm{erg} \mathrm{cm}^{-2} \mathrm{~s}^{-1}$, the fit is no longer valid as all of the data points lie at lower values than predicted by an extrapolation of the fit. Why do the more active stars violate the fit shown by the less active stars?

Before we apply the $\dot{M}-F_{X}$ relation to estimating the properties of the solar wind in time, it is important to understand the limitations of this relation. We have already mentioned that the mass loss and X-ray data are not simulataneous and that we have assumed that all of these stars have outflow velocities of $400 \mathrm{~km} \mathrm{~s}^{-1}$ similar to the quiet Sun. Also, the relation is based on data for stars with a range of spectral types (G2 V to $\mathrm{K} 5 \mathrm{~V}$ ), and some are binaries. Two of the more active stars are $\mathrm{M}$ dwarfs, but one is a G8 V star ( $\xi$ Boo). Clearly $\dot{M}$ values are needed for more stars to confirm the $\dot{M}$ $F_{X}$ relation for the less active stars and to determine whether the more active stars are inconsistent because they are more active or because they are M dwarfs. Nevertheless, eight $\mathrm{G}$ and K-type stars fit the relation within a factor of two despite the uncertainties, and we assume the relation to be valid as a working hypothesis. We have two approved $H S T / S T I S$ programs to observe eight more stars and there may be other observed targets to fill in the diagram and test our conclusions.

Ayres (1997) found a relation between X-ray surface flux and age $(t)$ for solar-type dwarf stars, $F_{X} \propto t^{-1.74 \pm 0.34}$. Combining this relation with the $\dot{M}-F_{X}$ relation leads to 

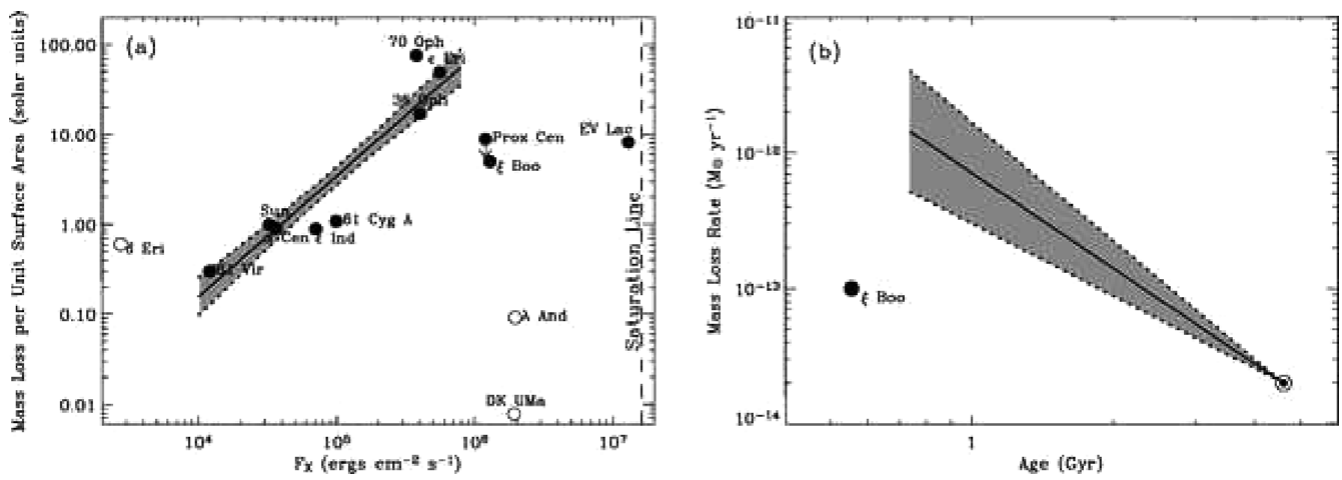

Figure 3. Left: Observed mass loss rates per $\mathrm{cm}^{2}$ of stellar surface are compared to X-ray surface fluxes. Filed circles are for main sequence stars and unfilled circles are for subgiants and giants. The solid and dashed lines are for Eq. 2.1 and its uncertainty. Right: solar mass loss rate from 700 Myr to the present computed from Eq. 2.2 (Wood et al. 2005).

$$
\dot{M} \propto t^{-2.33 \pm 0.55} \text {. }
$$

This relation shown in the right hand side of Fig. 3 provides an estimate of $\dot{\mathrm{M}}_{\odot}$ back to an age of $700 \mathrm{Myr}$ when $F_{X}$ would have been larger than the upper limit of the $\dot{\mathrm{M}}-F_{X}$ relation. This age is slightly larger than the age of the Hyades. The total mass loss for the Sun between $700 \mathrm{Myr}$ and the present is $0.001 M_{\odot}$ with an uncertainty of a factor of 10 (Wood et al. 2002). If $\dot{M}$ remained at $1.5 \times 10^{-12}$ ( \pm a factor of 3 ) $M_{\odot} \mathrm{yr}^{-1}$ over the Sun's early life on the main sequence $(0 \leqslant t \leqslant 700 \mathrm{Myr})$, then the additional mass loss would be $0.001 M_{\odot}$ ( \pm a factor of 3 ). This amount of mass loss would not have modified the Sun's evolution, but the amount of mass loss before 700 Myr is uncertain.

Can theoretical models for stars with solar-type magnetic field topologies explain the $\dot{\mathrm{M}}-F_{X}$ relation? Cranmer \& Saar (2011) find that Alfvén wave-driven winds predict a factor of 30 increase in $\dot{M}$ for a solar mass star as the rotation period decreases from 25 to six days, consistent with the increase in $\dot{\mathrm{M}}$ predicted by the $\dot{\mathrm{M}}-F_{X}$ relation.

Why is the $\dot{\mathrm{M}}-F_{X}$ relation not valid for the more active stars? A likely explanation is that the magnetic topology of rapidly rotating stars which have strong X-ray emission is different from that of slowly rotating stars. Zeeman-Dopler imaging studies show that rapidly rotating dwarf stars with mass greater than $0.5 \mathrm{M}_{\odot}$ have strong torroidal fields, and low mass stars often have strong poloidal fields (Donati \& Lanstreet 2009). The maximum $F_{X}$ for which Eq. 2.1 is valid corresponds to a rotation period of six days for a solar-mass star. Zeeman-Doppler images of such stars show large polar spots surrounded by torroidal magnetic fields. Theoretical models for stars with different magnetic topologies are needed to understand why the $\dot{M}-F_{X}$ relation is not valid for these stars. We note that the solar wind mass flux is nearly the same in coronal holes and equatorial regions despite the difference in magnetic field topology (Phillips 1995).

\section{References}

Ayres, T. R. 1997, J. Geophys. Res., 102, 1641

Baranov, V. B. \& Malama, Y. G. 1995, J. Geophys. Res., 100, 14755

Cranmer, S. R. \& Saar, S. H. 2011, ApJ, 741, 54

Donati, J.-F. \& Landstreet, J. D. 2009, ARAA, 47, 333

Izmodenov, V. V., Lallement, R., \& Malama, Y. G. 1999, A\&广A, 342, L13 
Müller, H.-R., Zank, G. P., \& Wood, B. E. 2001, ApJ, 551, 495

Phillips, J. L. 1995, Science, 268, 1030

Wood, B. E., Linsky, J. L., Müller, H.-R., \& Zank, G. P. 2001, ApJ Letters, 547, L49

Wood, B. E., Müller, H.-R., Zank, G. P., \& Linsky, J. L. 2005, ApJ, 574, 412

Wood, B. E., Müller, H.-R., Zank, G. P., Linsky, J. L., \& Redfield, S. 2005, ApJ Letters, 628, L143

Zank, G. P., Pauls, H. L., Williams, L. L., \& Hall, D. T. 1996, J. Geophys. Res., 101, 21,639

\section{Discussion}

DiBYENDU NANDI: We know that there is a strong power law relationship between magnetic flux and X-ray flux. Given that you find a connection between X-ray flux and stellar wind, perhaps this relationship can be utilized to infer the magnetic history of the Sun?

JEFFREY LiNSKY: Yes, we will look for a correlation of the unsigned average magnetic flux with the mass loss rate and then the magnetic field history of the Sun.

JANET LuHmann: Have you thought about the possible effect of a solar maximum type solar wind that is structured and bursty, rather than steady, laminar and uniform? Is your thinking affected by new IBEX results?

JefFrey Linsky: We find that the mass loss rate declines to a plateau when the Xray surface flux becomes greater that $10^{6} \mathrm{erg} \mathrm{cm}^{-2} \mathrm{~s}^{-1}$, which corresponds to a stellar rotation rate of about 7 days for a solar mass star. At this rotation rate the stellar magnetic field is probably more dipole-like than for the Sun. We have not considered bursty and inhomogeneous flows.

We look forward to IBEX measurements of the speed of in-flowing helium atoms from the interstellar medium. This will tell us whether the heliosphere is located inside the Local Interstellar Cloud (LIC) or in a transition region between the LIC and another cloud. The answer to this question will determine the outer boundary condition of the solar wind. 